\title{
The Use of Meta-Surfaces in Vehicular Networks
}

\author{
Barbara M. Masini ${ }^{1, *(\mathbb{D})}$, Cristiano M. Silva ${ }^{2}(\mathbb{D})$ and Ali Balador $^{3}(\mathbb{C})$ \\ 1 CNR-IEIIT, v.le Risorgimento, 2, 40136 Bologna, Italy \\ 2 Departamento de Tecnologia, Universidade Federal de São João del-Rei, São João del-Rei 36307-352, Brazil; \\ cristiano@ufsj.edu.br \\ 3 Malardalen University, 1, 72220 Vasteras, Sweden; ali.balador@ri.se \\ * Correspondence: barbara.masini@ieiit.cnr.it
}

Received: 30 November 2019; Accepted: 27 February 2020; Published: 2 March 2020

check for updates

\begin{abstract}
Mobility as a service is becoming a new paradigm in the direction of travel planning on the basis of the best service offered by the travelled roads. Hence, the environment in which people move will become smarter and more and more connected to grant services along the whole path. This opens new challenges related not only to the on board connectivity and wireless access technologies, but also on the reliability and efficiency of the surrounding environment. In this context, reconfigurable meta-surfaces play a crucial role, since they can be used to coat buildings, vehicles or any other suitable surfaces and let the environment become an active part of the communication system by opportunistically redirecting (i.e., reflecting, without generating new waves) signals to the target receivers. The objective of this paper is to highlight the limits of current wireless access technologies for vehicular scenarios and to discuss the potential impact of a smart environment made of reconfigurable meta-surfaces on some next generation vehicular use cases, such as cooperative driving and vulnerable road users (VRUs) detection. In addition, a preliminary model is presented to derive, in a simplified way, the performance of an IEEE 802.11p network in terms of collision probability. Even if analytical and based on simplified assumptions, this model has been validated through simulations and allows to compare the performance of the network with and without reconfigurable meta-surfaces.
\end{abstract}

Keywords: connected vehicles; reconfigurable meta-surface; smart environment; cooperative driving; vulnerable road user detection; collision probability

\section{Introduction}

Listening to Alexander Lautz from Deutsche Telekom, the device that, 10 years from now, we will look back at as the device of the 5G era, will be the car [1]. This is not just a catchphrase, but it is a clear index of the new perception of mobility as a service and not only as a transportation from an origin to a destination. Today, our on board smart navigator suggests the best route as the shortest one (in time or distance); tomorrow the mobility manager (on board or distributed in the cloud) will plan our commutes on the best connected roads or following the combination of routes and transportation that better fulfill the user's preference and needs. The concept of Internet of vehicles (IoV) has recently emerged just to indicate the need to go beyond the potentialities of vehicular ad-hoc networks (VANETs) and pass from the concept of smartphone to that of smart car, that is a moving connected object in a connected environment [2-5].

To obtain reliable service along the travel route, we need not only connected and automated car, but also a smart environment, able to opportunistically contribute to the signals propagation and to an ubiquitous and reliable coverage. 
Different wireless access technologies are running to come on the market of connected vehicles for vehicle-to-everything (V2X) communications [6,7]: on the one hand, the old fashioned IEEE 802.11p (or its European version ITS-G5) is only waiting for a mandatory and clear business model to be installed on board of all new vehicles [8,9] and, on the other hand, the newer cellular-V2X (C-V2X) proposed by 3GPP Release 14 is rushing into things promising better coverage, higher throughput and lower latency with respect to its competitor $[10,11]$. Recent works studied the performance of both IEEE 802.11p and C-V2X in different realistic scenarios, demonstrating their potentialities in terms of connectivity, packet reception ratio, latency and coverage, but also highlighting some limits in obstructed scenarios or congested roads [12-21].

What could be enhanced, looking forward, is the environment: a smart environment could drastically enhance the performance of wireless access technologies and having, as a consequence, an impact on connected vehicles related applications. Motivated by this, researchers from both the academical world and the industrial one, are proposing new solutions to smarten up the cities, starting from the street, buildings and citizen themselves [1]. This includes sensors embedded in the roadway [22], wireless access technologies on traffic lights or lamps along the roads [23], vehicular social networks [24], vehicles' routing [25-27], management of vehicular communication [28,29], cameras, smart wearable devices, etc. In this context, for example, the pilot project Austria's Autobahn uses Cisco's devices to connect tens thousands sensors with the objective to monitor traffic and road conditions. This represents an important example of how cities and public administrations move toward smart connected environments to improve safety, traffic efficiency, road capacity and infotainment $[30,31]$. The interest in smart environment is demonstrated also by the 6G Wireless Flagship Program (the world's first 6G research program), which indicates beside new wireless communication and computer science topics, also the importance of new electronics materials.

However, in spite of the huge effort in this direction, there will still scenarios in which the communication is obstructed by strong obstacles, preventing good links and allowing poor performance in terms of data rate, error rate, coverage and latency. In this context, reconfigurable meta-surfaces can play an active role, opportunistically redirecting the radio waves to improve connectivity and enabling the establishing of new and potentially stronger links [32]. Meta-surfaces are thin electromagnetic meta-material with typically sub-wavelength thickness and large in transverse size [33,34]. They are composed of sub-wavelength scattering particles that can revise the Snell's law redirecting the radio waves in the desired direction and can do this run time, changing the redirection of the waves time by time, according to the generalized Snell's laws, thus providing different values for the angles of incidence and reflection. Beyond meta-surface, what it is really challenging and stimulating, is the use of a reconfigurable meta-surface, where the scattering particles are not fixed, but can be moved and modified depending on the input they receive from the external world [35,36]. The idea of reconfiguring the wireless propagation environment has emerged only recently with focus especially on the indoor environments, where reconfigurable meta surfaces become connected to the rest of the scenario interacting with the connected objects and serving the user needs in unprecedented ways [37,38].

Several works deal with antenna design and performance optimization, such as [39], where a meta-superstrate for two vertically polarized MIMO antenna elements at the base-station is proposed to reduce the inter-element spacing. Preliminary evaluations related to the use of meta-surfaces in outdoor scenarios are related to the proposal of algorithms to minimize the total transmit power at the base station of a cellular system conditioned to the users quality of service (QoS) constraints [40] or to maximize either the energy or the spectral efficiency of a reconfigurable meta-surface multi-user MISO system [41]. Instead of using sensors embedded in the roadway and on traffic lights, reconfigurable meta-surfaces could be exploited to extend coverage in the highly dynamic vehicular environment, by coating the environment with intelligent meta materials. 
Hence, the objective of this work can be summarized as follows:

- $\quad$ To describe the main characteristics of meta-surfaces.

- To highlight potential uses of reconfigurable meta-surfaces especially when adopted in vehicular environments. In this context, two main use cases are considered: cooperative driving and vulnerable road users (VRUs) detection.

- To demonstrate, through a simple analytical model (validated by simulation), the improvement that a reconfigurable meta-surface can provide in reducing the collision probability when random access to the medium is adopted for vehicle-to-vehicle (V2V) communications.

This work is organized as follows: in Section 2, the main advantages and limits of the two main candidates radio access technologies for vehicular networks are highlighted; in Section 3, the concept of reconfigurable meta surface is introduced and the use of reconfigurable meta-surfaces in vehicular networks is proposed and discussed especially referring to two case studies, cooperative driving and pedestrian detection. A simplified model for the evaluation of the impact of meta-surfaces on the IEEE 802.11p performance in terms of collision probability is presented in Section 4 and is validated by simulations. Finally, in Section 5 our conclusions are drawn.

\section{Technologies for Vehicular Networks}

Nowadays, two are the candidate enabling technologies for vehicular communications: IEEE 802.11p (or its European version ETSI ITS G5) and C-V2X.

IEEE 802.11 p dates back in 2004 when the IEEE 802.11 working group started a discussion on how to modify and adapt Wi-Fi for dynamic environment, reducing the signaling and overhead of the nomadic version to support a completely different environments. It was then standardized in 2010 and tested in different field trials all around the world also with thousands of vehicles, demonstrating good performance in different use cases, thus representing a commercially available technology. On the other hand, C-V2X has been defined by 3GPP in 2016 within long term evolution (LTE) Release 14 and frozen in 2017 with the first plug test that took place in December 2019 in Malaga, Spain, demonstrating the $95 \%$ of success in terms of interoperability issues [42]. Hence, it can be observed that, while the IEEE $802.11 \mathrm{p}$ community spent several years from the first discussions to standardization and then on how and when set it up it on board, the cellular world sprinted forward and in a couple of years, test devices are ready for interoperability tests using a widespread technology, as it is demonstrated by the summary of timeline in Figure 1, with different colors showing the timeline of the different technologies. As it can be observed, the speed of development of C-V2X is much higher than Wi-Fi for mobility (IEEE 802.11p) and, despite being frozen in 2017, it will be ready for commercial installation on boards from 2020.

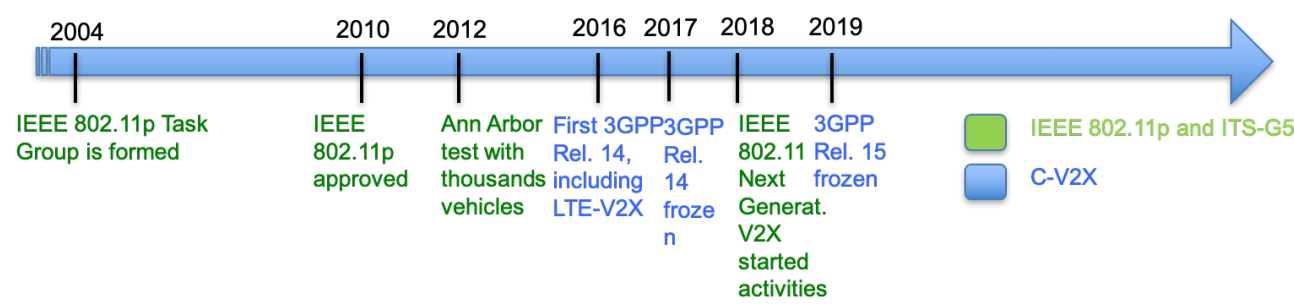

Figure 1. Summary of timeline of Wi-Fi for mobility and cellular vehicle-to-everything (C-V2X), showing the different sprint of the two standardization and experimentation processes.

\subsection{IEEE $802.11 p$}

IEEE 802.11p defines the physical (PHY) and medium access control (MAC) layer protocols. At the PHY layer, IEEE 802.11p adopts orthogonal frequency division multiplexing (OFDM) with 52 subcarriers of which 48 used for data and 4 for pilots. The OFDM symbol lasts $8 \mu$ s and the 
subcarrier spacing is $156.25 \mathrm{kHz}$, bringing to a raw bandwidth of $10 \mathrm{MHz}$. Eight modulation and coding schemes (MCSs) are possible, with modulations going from BPSK to 16-QAM with convolutional coding. At the MAC layer, carrier sensing multiple access with collision avoidance (CSMA/CA) is adopted, hence, when a node has to transmit a packet, it senses the medium; if the medium is idle the packet is transmitted after an Arbitration inter-frame spacing (AIFS) interval time (that takes into account potential delays in the propagation due to distant nodes), otherwise a mechanism based on random backoff is performed to reduce the probability of collisions by letting nodes to randomly start the next sensing phase for transmission. In addition, in the vehicular scenario, the acknowledgement and request to send/clear to send-RTS/CTS-mechanism are not foreseen to accelerate connection.

CSMA/CA has the advantage of being completely distributed and does not need any synchronization procedure, but, on the other hand, it suffers from collisions in dense vehicular environments [43], thus mechanisms to avoid overloads are necessary, such as decentralized congestion control (DCC) algorithm proposed by ETSI and SAE or new algorithm proposed in the literature [44], such as full duplex carrier sensing multiple access with collision detection (CSMA/CD) mechanism [45].

\section{2. $C-V 2 X$}

LTE Release 14 represents the first solution in the history of cellular world which includes direct resource allocation and communication without cellular coverage provided by an eNodeB with devices in high relative mobility. LTE-V2X is also called sidelink (since not downlink or uplink, but direct V2V) and its communication interface is named PC5. At the PHY layer, LTE-V2X adopts OFDM as IEEE 802.11p, whereas at the MAC layer it is based on the access used in the uplink of LTE, hence single carrier frequency division multiple access (SC-FDMA), that consists in a sort of pre-distorsion of the OFDM signal to behave as a single carrier in the presence of non linear effects. However, resources are orthogonally allocated in the frequency-time matrix as for orthogonal frequency division multiple access (OFDMA). Specifically, in the frequency domain, the subcarrier spacing is $15 \mathrm{kHz}$ and subcarriers are allocated in groups of 12 (i.e., $180 \mathrm{kHz}$ ); in the time domain, 14 symbols form a subframe of $1 \mathrm{~ms}$, also called transmission time interval (TTI), and include 9 data symbols, 4 demodulation reference signal (DMRS) symbols, and 1 empty symbol for Tx-Rx switch and timing adjustment. LTE-V2X has a high number of MCSs, with 4-QAM and 16-QAM modulations and an almost continuous coding rate.

Two different modes are defined by 3GPP for resources allocation, Mode 3 and Mode 4 [10]. In both modes communication is in direct (ad-hoc) mode between two vehicles, but they differ in the way resources are allocated. In Mode 3, also known as controlled allocation, the resources are defined and allocated by the network, hence, it can be adopted when vehicles are in the coverage of an eNodeB. In Mode 4, also known as autonomous or out-of-coverage allocation, each node selects the resources to use for communication based on a sensing procedure and an semi-persistent scheduling (SPS) mechanism [12,46], hence it is fully distributed as IEEE 802.11p.

\subsection{Challenges and Limits of Current Technologies}

Since Release 14 was defined, several studies have been carried out to compare IEEE 802.11p and C-V2X: many works push C-V2X as a more efficient technology, supported by an already deployed architecture diffused worldwide and a clearer road-map for future evolutions. The 5G Automotive Association (5GAA) pushes the 5th generation C-V2X to simplify the communication among the different road users, whereas the Car-to-Car Consortium (C2C-CO) claims that a largely tested and consolidated technology such as Wi-Fi for mobility could be the starting point for future vehicular communications. In [11], comparative experiments with real devices are shown, demonstrating longer ranges for C-V2X with respect to IEEE 802.11p and verifying that the latency in C-V2X under congested conditions does not exceed $100 \mathrm{~ms}$. Other works, instead, push IEEE 802.11p since it represents a widely tested and reliable technology, already available on the market [47]. 
IEEE 802.11p suffers from collisions due to random access to the channel. Since each node transmits only if the medium is sensed as idle after a random backoff interval time, collisions typically arise in dense scenarios when an increasing number of vehicles try to access the medium and transmit. This mechanism may cause a high resources wasting since a transmission needs to come to an end before a collision is recognized, even if the collision happened at the very beginning. Hence, the number of terminals hidden to each others increases, since two nodes transmitting to the same destination but are far from each other may not sense the reciprocal interference. Another problem is constituted by the exposed terminal problem that happens when two different transmissions toward two different destinations senses each other and defer the transmission even if they could occupy the channel.

Hence, IEEE $802.11 \mathrm{p}$ provides satisfactory performance for most vehicular applications (especially those typically requiring a latency around $100 \mathrm{~ms}$ ), when the vehicular density is moderate. When the vehicular density increases, IEEE $802.11 \mathrm{p}$ performance rapidly deteriorates due to packet collisions. Another limit to the diffusion of IEEE 802.11p is also the need of road side units (RSUs) development along the roads, but this is out of the scope of the present work [48].

The access is more robust in C-V2X networks, where orthogonal resources are allocated (by the eNodeB in Mode 3 or autonomously in Mode 4) to reduce interference to the minimum. Several works show that the performance of C-V2X sidelink Mode 4 is higher with respect to that of IEEE 802.11p in terms of signal to noise ratio (SNR) and coverage. However, when the traffic density increases, the performance of C-V2X, too, drops rapidly, particularly for Mode 4 resulting in an increased interference level among C-V2X users [12].

Given the fast development of C-V2X, also IEEE formed a new Task Group in January 2019, named IEEE $802.11 \mathrm{bd}$ to study the evolution of 802.11 for next-generation V2X communications. The attention was specifically focused on acOFDM numerology re-design, multiple input multiple output (MIMO) techniques, advanced channel coding, and better pilots placing, Dual carrier modulation (DCM) and $20 \mathrm{MHz}$ channels (hence, band doubled with respect to the actual release).

Also, 3GPP is working for next generation systems and in September 2019 Release 16 defined new architectural modification to support advanced V2X applications with more stringent QoS requirements compared to applications that can be supported by C-V2X. Release 16 is quite specific in defining use-cases requirements and allows new solutions that support a latency lower than $3 \mathrm{~ms}$ with a reliability of $99.999 \%$ [22,49]. The design of two different and contemporary wireless access technologies, if from the one hand presents regional regulators and auto-manufacturers with two options, on the other hand, it results in challenging spectrum management issues.

In addition to the technological limits of each technology, the wireless propagation environment in vehicular networks suffers from highly dynamical scenario, of sudden obstructions, complex intersections, etc. [50]. Hence, independently on which technology will be set up first on board, the environment still play a severe propagation role for vehicular connectivity, providing absence of control over the wireless propagation environment and, often, as a consequence, high power consumption of the wireless interface. It is in this context that reconfigurable meta-surfaces could help in reflecting or refracting the impinging waves opportunistically to improve the link QoS and reach a higher number of users/vehicles with a target key performance indicator (KPI).

\section{Reconfigurable Meta-Surfaces in Vehicular Scenarios}

\subsection{Reconfigurable Meta-Surfaces: Generalities}

A meta-surface is an artificial surface made by the repetition of an electromagnetic element, the meta-atom, to provide properties that cannot be found in natural materials. The major difference between a surface and a meta-surface relies in the properties of the latter of shaping the radio waves according to the generalized Snell's laws of reflection and refraction, providing a spatial phase variation with sub-wavelength resolution to control the direction of wave propagation and the shape of wavefront [51]. 
When meta-atoms are static, we have a static interaction with electromagnetic waves, but if meta-atoms incorporate phase switching components (such as MEMS or CMOS transistors), the scattering particles are not fixed and we can have that impinging waves are routed according to provide customized reflections and the scattering particles can be modified depending on the stimuli that the meta-surface receives from the external world. Hence, very small reflecting elements can be used to coat natural surfaces and smartly reconfigure the signal propagation for performance enhancement.

In [33], two different types of reconfigurable meta-surfaces are discussed: the first type is represented by coating surfaces for walls, buildings, etc., that can be managed by a network operator as software-defined radio, with the main objective to improve the network coverage; the second type is the one than can be embedded into objects, as for wearable devices for health monitoring, that can backscatter the impinging radio waves to relay the acquired information. In both cases, it is worth noting that reconfigurable meta-surfaces allow wireless network operators to offer new services without emitting additional radio waves, by simply recycling those already existing for other purposes.

The specific arrangements of the scattering particles determine how the meta-surface transforms the incident wave into arbitrary specified reflected and transmitted radio waves. As shown, for example, in Figure 2, the two vehicles cannot communicate neither through V2V communication, due to the presence of a truck made in large part of iron-based materials, nor through vehicle-to-infrastructure (V2I) communication due to high and large buildings reducing the link QoS to unacceptable levels. However, the presence of an additional element, coated with a reconfigurable meta-surface, allows the redirection of the waves to let the link between the two vehicles possible, transforming the environment from adversary to collaborator, improving the coverage and making the propagation conditions favorable. We can also think to the particles of the meta-surface as embedded antennas constituted by a planar array of a large number of reconfigurable passive elements, where each element is able to independently introduce a certain phase shift onto the incident electromagnetic waves.

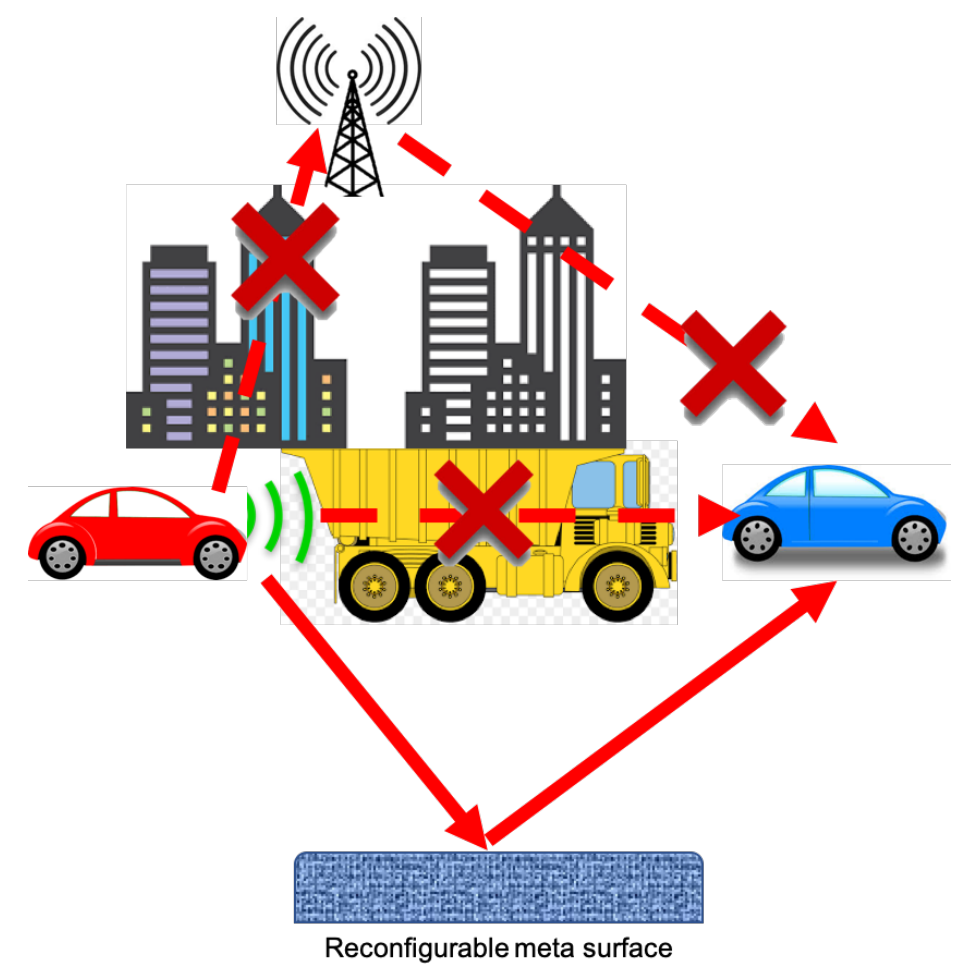

Figure 2. An opportune obstacle coated with meta material to redirect the electromagnetic waves toward the target receiver, which could not be reached through direct link due to an obstacle. 
This can yield extended coverage range with higher SNR levels with respect to the natural environment, in some cases also doubling the coverage distance with the same SNR level of natural environment [52].

In addition, reconfigurable meta-surfaces acting as reflectors are not affected by self-interference and by noise amplification effects since they do not act as relays and are not affected by such impairments [33]. However, beside their incredible properties, meta-surfaces also present some drawbacks. For example, the smart environment is more sensitive to channel estimation errors with performance that deteriorates much more than the classical system as the channel training SNR decreases. Moreover, the channel estimation errors are more significant as the user moves away from the reconfigurable meta-surface.

Another critical issue is constituted by the amount of sensed data that the meta-surfaces need to gather and reflect to be able to configure and optimize the environment following the network requirements. It has also to be observed that meta-surfaces reflect the impinging waves and the reflected rays are phase shifted and delayed. The phase shift and the delays introduced depend in a large part on the intrinsic factor of the surfaces, such as absorption and dielectric properties. Since latency is a critical requirement to satisfy, especially in next generation safety applications, these are characteristics that have to be taken into consideration during the engineering process to satisfy the QoS of a given environment [51]. Last, but not least, how to integrate reconfigurable meta-surfaces in future wireless networks is still an open issue.

Table 1 summarizes the main advantages and drawbacks or challenges of reconfigurable meta-surfaces.

In spite of these challenges, reconfigurable meta-surfaces play a crucial role in interconnecting the physical and the digital worlds in a seamless and efficient manner [33].

Table 1. Main advantages and challenges or drawbacks of reconfigurable meta-surfaces.

\begin{tabular}{ll}
\hline Pros & Cons \\
\hline $\begin{array}{l}\text { Enlarge the concept of software } \\
\text { networks }\end{array}$ & $\begin{array}{l}\text { Only prototypes are currently } \\
\text { available }\end{array}$ \\
\hline $\begin{array}{l}\text { No generation of new signals but } \\
\text { reuse of existing ones }\end{array}$ & $\begin{array}{l}\text { Not immediate integration in } \\
\text { wireless networks }\end{array}$ \\
\hline Programmable frequency selection & $\begin{array}{l}\text { Performance of wireless networks } \\
\text { with meta-surfaces still under } \\
\text { investigation }\end{array}$ \\
\hline $\begin{array}{l}\text { Potential increasing of information } \\
\text { reliability }\end{array}$ & $\begin{array}{l}\text { More sensitive to channel estimation } \\
\text { errors }\end{array}$ \\
\hline Sensing capabilities & $\begin{array}{l}\text { Potential need of power sources } \\
\text { Storage capabilities }\end{array}$ \\
\hline Deployment scalability & $\begin{array}{l}\text { Can introduce delays by storing and } \\
\text { releasing the reflected signals }\end{array}$ \\
\hline $\begin{array}{l}\text { Can offer new services without } \\
\text { emitting additional radio waves }\end{array}$ & $\begin{array}{l}\text { Reflected waves are phase shifted } \\
\text { and delayed }\end{array}$ \\
\hline
\end{tabular}

\subsection{Reconfigurable Meta-Surfaces for Enhanced Vehicular Scenarios}

The propagation environment in vehicular networks is constituted by a set of physical fixed and mobile objects that affect the propagation of electromagnetic waves between the communicating devices, often causing detrimental effects on the communication process. It is outside the network control. Software reconfigurable meta-surfaces (obtained, for example, by coating the walls of the buildings between the communicating devices) can mitigate the negative effect of the propagation environment by controlling the electromagnetic behavior of reflecting and refracting waves according 
to the generalized Snell's law, i.e., the angles of incidence and reflection of the radio waves can be different and depend on the phases induced by the elements of the reconfigurable meta-surface [52].

Looking, for example, at Figure 3, two vehicles approaching a highly obstructed intersection from two different directions (south and east in Figure 3a), could not be aware one of each other. But, if the buildings were coated with reconfigurable meta surfaces remote programmable as in Figure $3 b$, the electromagnetic waves could be opportunistically refracted toward the desired target receiver, improving safety applications, both in terms of link QoS and latency.

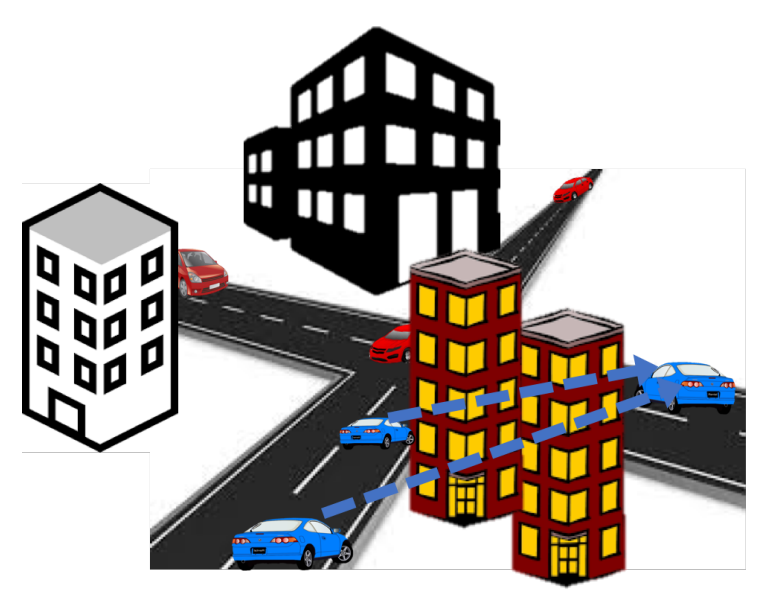

(a) Buildings with natural surfaces.

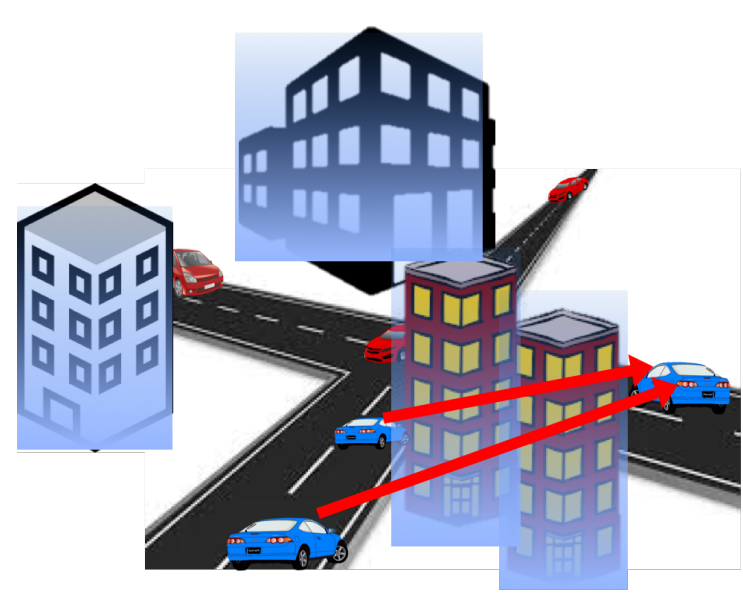

(b) Buildings coated with reconfigurable meta-surfaces.

Figure 3. The impact of meta-surfaces in obstructed intersections.

In Table 2, the possible uses of reconfigurable meta-surfaces are listed and some enabled or improved applications in vehicular scenarios are summarized.

Table 2. Potential uses of reconfigurable meta-surfaces when applied to vehicular applications.

\begin{tabular}{lll}
\hline $\begin{array}{l}\text { Potential Uses of } \\
\text { Reconfigurable } \\
\text { Meta-Surfaces }\end{array}$ & Safety & Non-Safety \\
\hline Beamforming & $\begin{array}{l}\text { Incident detection } \\
\text { Hazardous warning } \\
\text { Cooperative collision avoidance } \\
\text { Trajectories alignment }\end{array}$ & Video Sharing \\
\hline Range extension & $\begin{array}{l}\text { Information sharing for automated driving } \\
\text { Precise long horizon information } \\
\text { Cooperative platooning } \\
\text { Cooperative driving }\end{array}$ & Info-traffic sharing \\
\hline Uplink bottleneck & Remote driving information uploading & $\begin{array}{l}\text { Traffic information uploading } \\
\text { resolution for V2I }\end{array}$ \\
\hline Positioning & $\begin{array}{l}\text { V2R detection } \\
\text { V2P communication }\end{array}$ & $\begin{array}{l}\text { Personalized information } \\
\text { Location aware information }\end{array}$ \\
\hline Remote sensing & Hazard prevention & $\begin{array}{l}\text { Environment detection } \\
\text { Extended sensors }\end{array}$ \\
\hline
\end{tabular}

In the following, we consider two main case studies where reconfigurable meta surfaces could provide important performance improvements: cooperative driving and pedestrian detection. 


\subsection{Cooperative Driving}

Cooperative driving is listed among the challenging future case studies that next wireless access technologies have to address. Basically, actual applications that could be already available through IEEE 802.11p or LTE-V2X communication systems, are represented by the so called Day 1 and Day 1.5 cooperative-ITS service list [53] and are mostly based on the exchange of periodic beacon messages among vehicles to enable the awareness of the environment (e.g., probe vehicle data, traffic information, smart routing, etc.). In the future, vehicles will not only rely on information exchange related to actual position, speed, acceleration, etc., but they will also need to share intentions: this will allow each vehicle to have a glimpse into the future of other vehicles, allowing a human driver or an artificial on board intelligence to take the best decision/behavior.

5GCAR has identified five relevant use case classes: cooperative maneuver, cooperative perception, cooperative safety, autonomous navigation, remote driving [1]. Focusing on cooperative maneuver, the principle is, from the one hand, the sharing of local awareness and driving intentions and, on the other hand, the negotiation of the planned trajectories. This way, the driving trajectories can be coordinated and even optimized in a centralized or decentralized manner. In summary, cooperative driving systems are based on algorithms that control the vehicle behavior based on the behavior of the surrounding vehicles and allow to extend the perception range beyond line-of-sight and sensing angles. Examples of cooperative maneuver are cooperative lane merging, cooperative intersection crossing, platooning, etc. [54].

Today, for example, the $75 \%$ of lane changing accidents occur because of a lack of perception of the surrounding environment [55]. IEEE 802.11p and C-V2X Release14 cannot fully address cooperative driving requirements in terms of latency and reliability C-V2X Release 15 will incorporate 5G new radio (NR) features, providing higher data rate, ultra-low latency and higher reliability so that advanced use cases, such as cooperative driving, can be adddressed. In addition, accurate positioning and ranging will be included to enable sub-meter positioning.

To enable cooperative driving, information coming from different sensors of different vehicles of different vendors should be properly merged in a fast and reliable manner and each information should be strictly related to its accurate position in time and space [56]. This also implies that, as more and more advanced sensors populate the cars and produce information to be exchanged for a reliable cooperation, the amount of information to be exchanged increases and the risk of errors on the transmission channel increases as well [57]. Hence, new approaches have to be conceived. An example of fully distributed cooperation system for lane merging is reported in Figure 4, highlighting the impact of an obstructing building. In case it is coated with reconfigurable meta-surface, the probability of correct packet reception between the two vehicles would result increased. This scenario implies that all vehicles are equipped with sensing capabilities, data processing units also including data merging and data fusion and artificial intelligent capabilities for decision making.

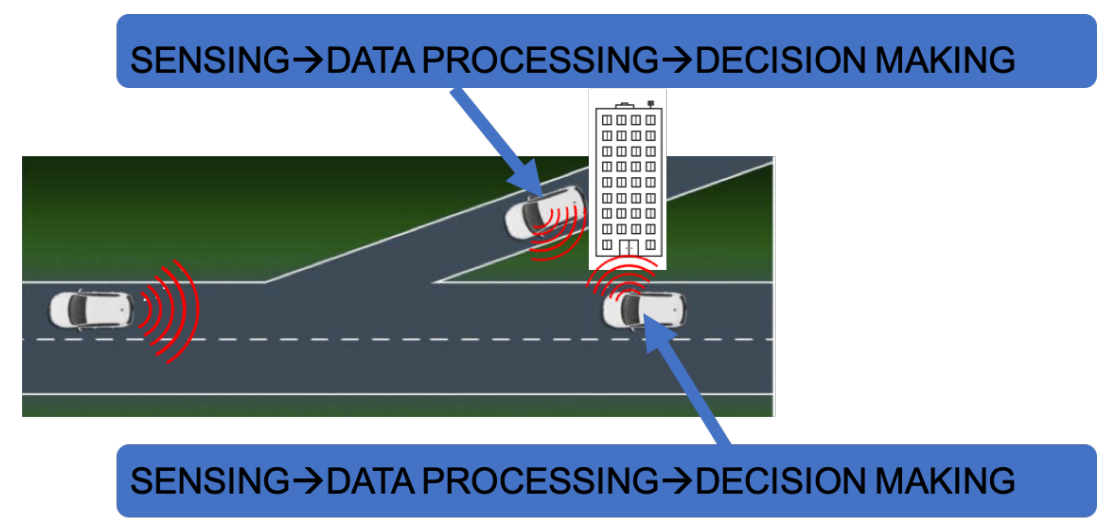

Figure 4. Example of cooperative driving scenario: lane merging. 


\subsection{Pedestrian Detection}

In a document released by the European Commission in Brussels on 2019 April 4, it is possible to read that the European Union has some of the safest roads in the world. Nevertheless, more than 25,000 people still lose their lives on EU roads every year, and many more are seriously injured. In 2018, around 25,100 road fatalities were reported by the $28 \mathrm{EU}$ Member States. This number is still very high, but it represents a first decrease of $21 \%$ compared to 2010.

A report provided by the National Highway Traffic Safety Administration (NHTSA) shows that over the past 40 years there has been a general downward trend in road mortal accidents, but focusing on the VRUs, in the year 2018, in the US 6283 pedestrians were killed, which is 3.4\% higher that in 2017 and the highest number since 1990. Hence, in spite of safety measurements taken worldwide to reduce fatalities and injuries have lowered the number of accidents, these solutions do not protect the VRUs [58].

Hence, in addition to the safety measures to protect vehicle occupants, determined efforts have to be done to implement specific measures to prevent fatalities and injuries of VRUs, such as cyclists and pedestrians, is needed to protect users outside of the vehicle [59]. Current pedestrian detection is based on advanced driver assistance systems (ADAS) with onboard sensors, such as cameras, radar and LIDAR, to detect the presence of VRUs [60,61]. All of these sensors require line of sight (LOS) to properly work and they cannot detect VRUs in case of obstacles partially or totally occluding the visibility, such as trees, trucks or buildings.

The importance and actuality of this issue is also demonstrated by the fact that the U.S. Department of Transportation (USDOT) has released a summary of the available vehicle-to-pedestrian (V2P) technologies. Among these technologies, alert/notification to users (e.g., through mobile phone alert) is the only connected way considered. Several works have been published in the last years on this topic; some of these works, like [62,63], consider a IEEE 802.11p V2P communication, demonstrating a probability to inform the VRU around 0.8 with a beacon generation frequency at least equal to $1 \mathrm{~Hz}$ (for IEEE 802.11p, a unique Provider Service Identifier (PSID) to identify applications for the safety of VRUs is defined by standard [64]).

However, as stated in Section 2.3, IEEE 802.11p-based V2X communication suffers from congestions on the medium when the number of vehicles (and VRUs) increases. In urban centers, for examples, VRUs are present in large numbers leading to dense networks, whose performance quickly decreases. Adult VRUs have a smartphone in their pocket and, today, this is the easiest way to provide an alert. However, the actual embedded cellular technology (4G) does not allow V2X communication, yet, but only communications through the eNodeB, thus with a latency which could be critical for VRUs' safety applications [65]. When V2P communication is addressed, another important aspect to be carefully considered is positioning, since the global positioning system (GPS) accuracy and the accuracy of the combination of different sensors, even if suitable for different ranges and weather conditions, is not sufficient [66,67]. Actual cellular positioning also provides poor accuracy, but this could be (at least partially) overcome with the introduction of 5G wireless communication systems introducing MIMO systems at mmWave [68]. However, mmWave suffers from limited coverage ranges and are deeply attenuated by obstacles, thus they can help only in specific scenarios and typically short distances.

Therefore, new approaches are necessary trying to ensure the reliable delivery of safety messages. Figure 5 shows an example of how reconfigurable meta-surfaces can contribute to redirect messages to warn pedestrians hidden to an incoming vehicle. Note, in fact, that, in this case, radio communication can be drastically obstructed, but the coated building can however provide the timely delivery of the warning message.

Another issue to be addressed is related to the limited battery life of smartphones when used for V2P communications. In fact, in order to prevent accidents, both pedestrian smartphones and on board units (OBUs) need to frequently transmit beacons containing at least position, speed and direction. These periodic messages, even when sending small amount of data, can drain the smartphone 
battery very quickly $[69,70]$. This issue could be overcome, for example, with $\mathrm{t}$-shirts coated with a reconfigurable meta-surface that, instead of transmitting a new signal consuming energy, simply reflects the received wave. It is also worth noting that some VRUs could not be connected at all, such as children. Hence, the protection and detection of VRUs represent another challenging and important scenario where a smart environment can act toward the improvement of safety. In fact, meta-surfaces can not only extend the coverage range, but also perform redirecting toward the right obstacle, alerting in case of real necessity.

\section{The Impact of Meta-Surfaces: Performance Example}

To provide numerical examples on the efficiency of meta-surfaces, we focus, in this Section, on IEEE 802.11p as enabling technology. To access the medium, IEEE 802.11p adopts CSMA/CA, hence, when a node needs to transmit a packet, it starts listening to the channel for an AIFS period, after which, if the channel is sensed idle, the packet is transmitted. If during AIFS the channel is sensed as busy or becomes busy, a random backoff algorithm is applied and the node will try to access the channel again after a random interval time chosen in the range [0-CW] [71]. Given this mechanism, IEEE 802.11p performance suffers from the hidden terminal effect and capture effect. As anticipated in Section 2.1, two nodes are hidden from each other when they are out of the reciprocal sensing range, but they are both transmitting toward the same destination. The capture effect, instead, is due to the presence of multiple transmitters that are sending their messages at the same time towards the same destination; in this case, it may happen that the power level of one of them is sufficiently higher than the interference received from the others to allow correct decoding at the receiver. Otherwise, the message is not captured.

To make some example evaluations, we assume a highway scenario with variable traffic conditions and multiple lanes, as shown in Figure 6. To analytically estimate the collision probability, this 2-D scenario is approximated with an 1-D scenario with the assumption of Poisson distributed vehicles with variable density $\rho$. Each vehicle is assumed to be equipped with an OBU that periodically transmits beacons of $B_{\mathrm{b}}$ bytes with a frequency $f_{\mathrm{b}}$ expressed in $\mathrm{Hz}$.

Following the model presented in [15], it is possible to evaluate the probability to collide, either in visibility or in case of hidden terminals and not captured, as

$$
p_{\mathrm{c}}=1-\left(1-p_{\mathrm{c}-\mathrm{vis}}\right) \cdot\left(1-p_{\mathrm{c}-\mathrm{ht}} \cdot\left(1-\frac{p_{\mathrm{capt}}}{2}\right)\right)
$$

where $p_{\mathrm{c} \text {-vis }}$ is probability that the considered vehicle (i.e., an $\mathrm{OBU}$ ) senses the channel busy and ends with colliding with at least one other vehicle, $p_{\text {capt }}$ is the capture effect probability (the capture effect avoids the collision if and only if the interference received from the other transmitting node is below a given threshold) and $p_{\text {c-ht }}$ is the probability to collide due to the presence of an hidden terminal either ending or starting its transmission during the observed communication (more details to derive the different probabilities can be found in [15]).

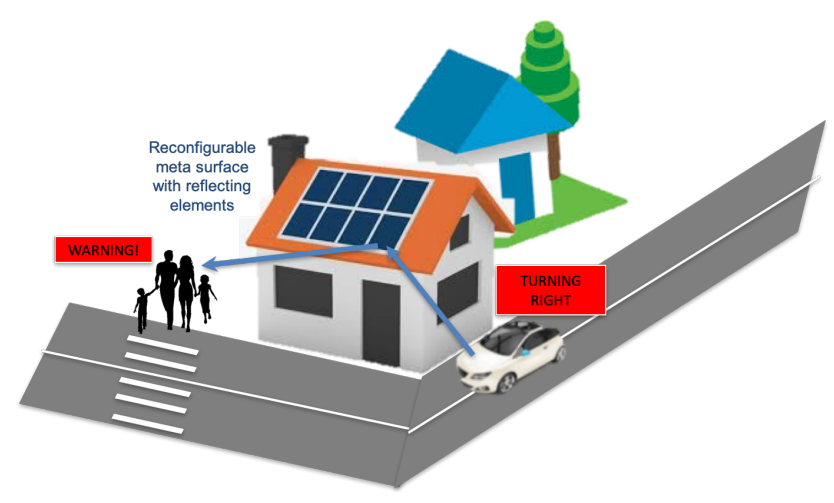

Figure 5. Pedestrian detection. 
In general (i.e., without meta-surfaces), the probability that a collision occurs due to the presence of hidden terminals can be found as [15]:

$$
p_{\mathrm{c}-\mathrm{ht}}=1-p_{\mathrm{nc}-\mathrm{h} 1} \cdot p_{\mathrm{nc}-\mathrm{h} 2}
$$

where $p_{\text {nc-h1 }}$ is the probability that no hidden terminal is transmitting and $p_{\text {nc-h2 }}$ is the probability that no hidden node starts its transmission during the observed communication.

Let us now assume that $p_{\text {c-vis }}$ and $p_{\text {capt }}$ remain equal also in the presence of meta-surfaces, whereas $p_{\mathrm{c} \text {-ht }}$ is affected by the presence of meta-surfaces which could limit the hidden terminal effect through the redirection of reflected paths that could extend the coverage, as shown in Figure 6. Hence, we assume the presence of at least one meta-surface coated object which allows to grant that no hidden node starts its transmission during the observed communication, always providing $p_{\mathrm{nc}-\mathrm{h} 2}=1$, simplifing (2) that reduces to

$$
p_{\mathrm{c}-\mathrm{ht}}=1-p_{\mathrm{nc}-\mathrm{h} 1} \text {. }
$$

Hence, the collision probability (1) becomes

$$
p_{\mathrm{c}}=1-\left(1-p_{\mathrm{c}-\mathrm{vis}}\right) \cdot\left[1-\left(1-p_{\mathrm{nc}-\mathrm{h} 1}\right) \cdot\left(1-\frac{p_{\text {capt }}}{2}\right)\right] .
$$

This is possible through the extension of the coverage range of the transmitting node that allows the hidden terminal to hear for an ongoing transmission, as shown in Figure 6b. This is a simplified model, but help in individuating and evaluating the impact of the proposed solution on the wireless access technology performance.

In order to provide some numerical results, we assume each vehicle equipped with an OBU that periodically transmits beacons of $B_{\mathrm{b}}=300$ bytes every $f_{\mathrm{b}}=10 \mathrm{~Hz}$. Among the eight available modes of IEEE 802.11p, we consider mode 3, providing a raw data rate of $6 \mathrm{Mb} / \mathrm{s}$.

To demonstrate the validity of the model simulation results are also provided, referring to a $16 \mathrm{~km}$ highway with 3 lanes per direction. The main settings are reported in Table 3. As far as the propagation is concerned, we assume the following path loss model

$$
L(x)=L_{0} \cdot x^{\beta}
$$

where $L_{0}$ is the path loss at the reference distance of $1 \mathrm{~m}, x$ is the distance between the transmitter and the receiver, and $\beta$ is the path loss exponent. Hence, given a distance $\mathrm{d}$ between the transmitting and receiving vehicles and $n_{\text {int }}$ interferers each at distance $d_{\text {int }}^{(i)}$ (with $i \in\left[1, n_{\text {int }}\right]$ ) from the receiver, all of them transmitting with the same power $P_{\mathrm{tx}}$, the signal to noise and interference ratio (SINR) at the receiver can be calculated calculated as

$$
\gamma=\frac{\frac{P_{\mathrm{tx}} \cdot G_{\mathrm{r}}}{L_{0} \cdot d^{\beta}}}{P_{\mathrm{n}}+\sum_{i \in\left[1, n_{\mathrm{int}}\right]} \frac{P_{\mathrm{tx}} \cdot G_{\mathrm{r}}}{L_{0} \cdot d_{\mathrm{int}}^{(i)}}}
$$

where $G_{\mathrm{r}}$ is the antenna gain at the receiver and $P_{\mathrm{n}}$ is the noise power. By inverting (6), the transmission range $r_{t x}$ (i.e., without interference) can be obtained as

$$
r_{\mathrm{tx}}=\left[\frac{P_{\mathrm{tx}} \cdot G_{\mathrm{r}}}{L_{0} \cdot P_{\mathrm{n}} \cdot \gamma}\right]^{1 / \beta}
$$

Vehicles in the transmission range $r_{\mathrm{tx}}$ that are not visible to the transmitter are considered as hidden terminals. 


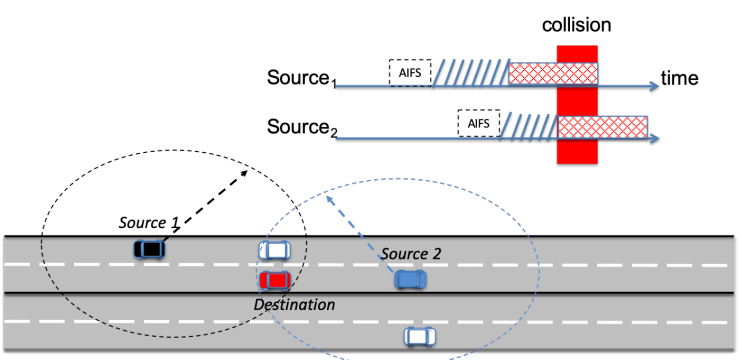

(a) Hidden terminal problem.

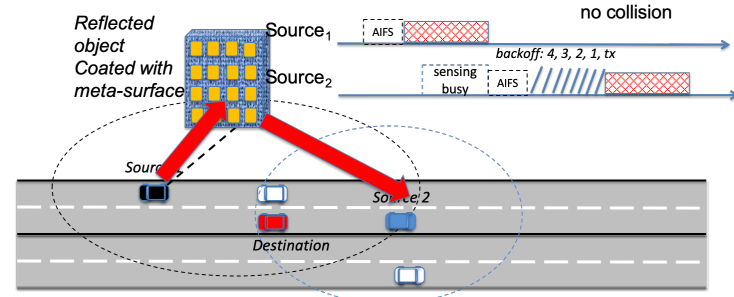

(b) The coverage range of Source ${ }_{1}$ is extended by a meta-surface and Source 2 senses the channel occupied and defers its transmission.

Figure 6. The coverage range of Source 1 can be extended by a meta-surface to solve the hidden terminal problem.

Table 3. Main settings.

\begin{tabular}{ll}
\hline Parameter (Symbol) & Value \\
\hline IEEE 802.11p mode & 3 \\
Modulation & QPSK \\
Coding rate & $1 / 2$ \\
Raw data rate & $6 \mathrm{Mb} / \mathrm{s}$ \\
Vehicle density $(\rho)$ & Variable \\
Beacon frequency $\left(f_{\mathrm{b}}\right)$ & $10 \mathrm{~Hz}$ \\
Vehicle density $(\rho)$ & Variable \\
Equivalent radiated power $\left(P_{\mathrm{tx}}\right)$ & $33 \mathrm{dBm}$ \\
Receiver antenna gain $\left(G_{\mathrm{r}}\right)$ & $3 \mathrm{~dB}$ \\
Path loss at 1 m at $5.9 \mathrm{GHz}\left(L_{0}\right)$ & $47.86 \mathrm{~dB}$ \\
Path loss exponent $(\beta)$ & 2.75 \\
Noise power over $10 \mathrm{MHz}\left(P_{\mathrm{n}}\right)$ & $-95 \mathrm{dBm}$ \\
Beacon size $\left(B_{\mathrm{b}}\right)$ & $300 \mathrm{bytes}$ \\
Contention window $(C \hat{W})$ & 15 \\
Slot duration $\left(t_{\sigma}\right)$ & $13 \mu \mathrm{s}$ \\
AIFS duration $\left(t_{\text {aifs }}\right)$ & $58 \mu \mathrm{s}$ \\
Overhead per packet & $40 \mu \mathrm{s}$ \\
\hline
\end{tabular}

In Figure 7, the collision probability given by (4) is plotted as a function of the distance for different values of vehicular density $\rho$. A comparison between the cases with and without meta-surfaces is reported. In addition, simulation results are also provided showing a good agreement with the analysis. As expected, independently on the vehicles density and on the use of meta-surfaces, the collision probability increases with the distance: for short distances the impact of hidden terminals is negligible and the collision probability is almost constant, then the impact of hidden terminals and low visibility is higher and the collision probability increases.

As a consequence, the impact of the use of meta-surfaces on the collision probability is much more evident as the distance and the vehicles density increase. For example, when the vehicles density $\rho=0.05$, the adoption of meta-surfaces can reduce the collision probability from 0.12 to around 0.06 at a distance of $350 \mathrm{~m}$, whereas when $\rho=0.2$, the collision probability has a higher impact, reducing the collision probability of around $42 \%$ from 0.41 to around 0.27 , which is a more tolerable value.

Note that since meta-surfaces do not act as relays, they do not introduce new signals in the network, hence the level of congestion is not increased. They reflect signals already present in the network, extending their coverage. In this sense, interference could also be extended, but algorithms to cancel it or reduce it could be applied as in other software-defined networks. 


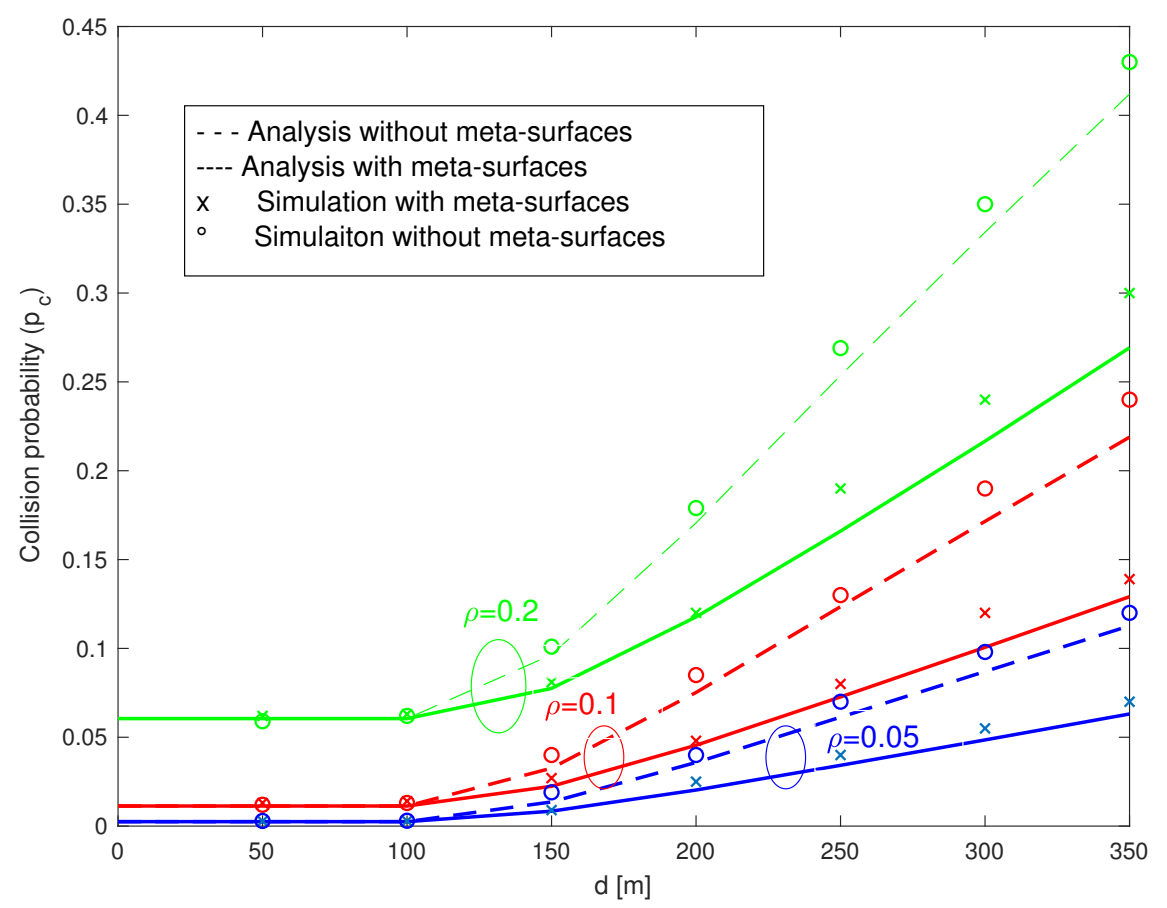

Figure 7. Collision probability as a function of the inter-vehicular distance with and without meta-surfaces varying the vehicles density $\rho$. Lines correspond to analysis, symbols to simulations.

\section{Consclusions}

In this work, we provided a general overview on how reconfigurable meta-surfaces can be used in vehicular scenarios by partially covering the limits of current wireless access technologies, such as IEEE 802.11p and C-V2X. Specifically, we aimed at underlining the main characteristics and potentialities of meta-surfaces when used in vehicular scenario, also providing a simple evaluation of their impact on network performance and demonstrating, in a simple scenario with Poisson random distributed vehicles, that if meta-surfaces can coat at least part of the scenario, they can extend the coverage range, limiting, for example, the hidden terminal problem that affects random access technologies. This model has been also validated by simulations on highways. Since meta-surfaces do not act as relays and do not introduce new signals in the network, the network congestion level is not affected and neither self interference nor amplification noise are added to the system.

Author Contributions: Conceptualization, methodology, software and writing original draft preparation: B.M.M.; literature review, writing review and editing, A.B. and C.M.S. All authors have read and agreed to the published version of the manuscript.

Funding: This research was partially funded by CNPq (Conselho Nacional de Desenvolvimento Científico e Tecnológico) grant 303933/2017-8, CAPES (Coordenação de Aperfeiçoamento de Pessoal de Nível Superior), and FAPEMIG (Fundação de Amparo à Pesquisa do Estado de Minas Gerais) grant APQ-02145-18.

Acknowledgments: The authors would like to thank Alessandro Bazzi and Alberto Zanella for helpful discussions and support, Marco di Renzo for his highlighting introduction to meta surfaces and Ian Akyildiz for his inspiring keynotes and never ending motivation.

Conflicts of Interest: The authors declare no conflict of interest.

\section{References}

1. On the Road to Self-Driving Cars, 5G Will Make Us Better Drivers. Available online: https: / spectrum.ieee.org/ telecom/wireless / mwc-barcelona-2019-on-the-road-to-selfdriving-cars-5g-will-make-us-better-drivers (accessed on 1 March 2020). 
2. Yang, F.; Wang, S.; Li, J.; Liu, Z.; Sun, Q. An overview of Internet of Vehicles. China Commun. 2014, 11, 1-15. [CrossRef]

3. Kaiwartya, O.; Abdullah, A.H.; Cao, Y.; Altameem, A.; Prasad, M.; Lin, C.; Liu, X. Internet of Vehicles: Motivation, Layered Architecture, Network Model, Challenges, and Future Aspects. IEEE Access 2016, 4, 5356-5373. [CrossRef]

4. Aliyu, A.; Kaiwartya, O.; Cao, Y.; Lloret, J.; Aslam, N.; Usman, M. Towards Video Streaming in IoT Environments: Vehicular Communication Perspective. Comput. Commun. 2017. [CrossRef]

5. Kumar, S.; Singh, K.; Kumar, S.; Kaiwartya, O.; Cao, Y.; Zhou, H. Delimitated Anti Jammer Scheme for Internet of Vehicle: Machine Learning Based Security Approach. IEEE Access 2019, 7, 113311-113323. [CrossRef]

6. Silva, C.M.; Masini, B.M.; Ferrari, G.; Thibault, I. A Survey on Infrastructure-Based Vehicular Networks. Mob. Inf. Syst. 2017, 2017, 28-56. [CrossRef]

7. Silva, C.M.; Silva, L.D.; Santos, L.A.L.; Sarubbi, J.F.M.; Pitsillides, A. Broadening Understanding on Managing the Communication Infrastructure in Vehicular Networks: Customizing the Coverage Using the Delta Network. Future Internet 2018, 11, 1. [CrossRef]

8. EUROPEAN COMMISSION. COMMISSION DELEGATED REGULATION (EU) of 13.3.2019 Supplementing Directive 2010/40/EU of the European Parliament and of the Council with Regard to the Deployment and Operational Use of Cooperative Intelligent Transport Systems. 2019. Available online: https:/ / spectrum.ieee.org/telecom/wireless/mwc-barcelona-2019-on-the-road-to-selfdriving-cars-5g-willmake-us-better-drivers (accessed on 1 March 2020).

9. Autotalks and NXP Semiconductors. IEEE 802.11p Ahead of LTE-V2V for Safety Applications. 2017. Available online: https:/ / www.nxp.com/docs/en/white-paper/LTE-V2V-WP.pdf (accessed on 1 March 2020).

10. Bazzi, A.; Cecchini, G.; Menarini, M.; Masini, B.M.; Zanella, A. Survey and Perspectives of Vehicular Wi-Fi versus Sidelink Cellular-V2X in the 5G Era. Future Internet 2019, 11, 122. [CrossRef]

11. 5GAA. V2X Functional and Performance Test Report; Test Procedures and Results. 2018. Available online: https://5gaa.org/wp-content/uploads/2018/10/FCC-USDOT-CV2X-v2.14_wo_Video-c1_Final.pdf (accessed on 1 March 2020).

12. Molina-Masegosa, R.; Gozalvez, J. LTE-V for Sidelink 5G V2X Vehicular Communications: A New 5G Technology for Short-Range Vehicle-to-Everything Communications. IEEE Veh. Technol. Mag. 2017, 12, 30-39. [CrossRef]

13. Min, W.; Winbjork, M.; Zhang, Z.; Blasco, R.; Do, H.; Sorrentino, S.; Belleschi, M.; Zang, Y. Comparison of LTE and DSRC-Based Connectivity for Intelligent Transportation Systems. In Proceedings of the IEEE 85th Vehicular Technology Conference (VTC Spring), Sydney, Australia, 4-7 June 2017; doi:10.1109/VTCSpring.2017.8108284. [CrossRef]

14. Nguyen, T.V.; Shailesh, P.; Sudhir, B.; Kapil, G.; Jiang, L.; Wu, Z.; Malladi, D.; Li, J. A Comparison of cellular vehicle-to-everything and dedicated short range communication. In Proceedings of the IEEE Vehicular Networking Conference (VNC), Torino, Italy, 27-29 November 2017.

15. Bazzi, A.; Masini, B.M.; Zanella, A.; Thibault, I. On the Performance of IEEE 802.11p and LTE-V2V for the Cooperative Awareness of Connected Vehicles. IEEE Trans. Veh. Technol. 2017, 66, 10419-10432. [CrossRef]

16. Cecchini, G.; Bazzi, A.; Masini, B.M.; Zanella, A. Performance comparison between IEEE 802.11p and LTE-V2V in-coverage and out-of-coverage for cooperative awareness. In Proceedings of the IEEE Vehicular Networking Conference (VNC), Torino, Italy, 27-29 November 2017; pp. 109-114. [CrossRef]

17. Vukadinovic, V.; Bakowski, K.; Marsch, P.; Garcia, I.D.; Xu, H.; Sybis, M.; Sroka, P.; Wesolowski, K.; Lister, D.; Thibault, I. 3GPP C-V2X and IEEE 802.11p for Vehicle-to-Vehicle communications in highway platooning scenarios. Ad Hoc Netw. 2018, 74, 17-29. [CrossRef]

18. Thota, J.; Abdullah, N.F.; Doufexi, A.; Armour, S. Performance of Car to Car Safety Broadcast Using Cellular V2V and IEEE 802.11P. In Proceedings of the IEEE Vehicular Technology Conference (VTC Spring), Porto, Portugal, 3-6 June 2018; pp. 1-5. [CrossRef]

19. Anwar, W.; Kulkarni, K.; Augustin, T.R.; Franchi, N.; Fettweis, G. PHY Abstraction Techniques for IEEE 802.11p and LTE-V2V: Applications and Analysis. In Proceedings of the 2018 IEEE Globecom Workshops (GC Wkshps), Abu Dhabi, UAE, 9-13 December 2018; pp. 1-7. [CrossRef] 
20. Kühlmorgen, S.; Schmager, P.; Festag, A.; Fettweis, G. Simulation-based Evaluation of ETSI ITS-G5 and Cellular-VCS in a Real-World Road Traffic Scenario. In Proceedings of the IEEE Vehicular Technology Conference (VTC Fall), Chicago, IL USA, 27-30 August 2018.

21. Bastos, A.V.; Silva, C.M.; Silva, D., Jr. Assisted Routing Algorithm for D2D Communication in 5G Wireless Networks. In Proceedings of the 2018 Wireless Days (WD) (WD'18), Dubai, UAE, 3-5 April 2018; doi:10.1109/WD.2018.8361688. [CrossRef]

22. Silva, C.M.; Pitangui, C.G.; Miguel, E.C.; Santos, L.A.; Torres, K.B. Gamma-Reload Deployment: Planning the communication infrastructure for serving streaming for connected vehicles. Veh. Commun. 2020, 21, 100197. [CrossRef]

23. Silva, C.M.; Aquino, A.L.L.; Meira, W., Jr. Smart Traffic Light for Low Traffic Conditions. Mob. Netw. Appl. 2015, 1-9. [CrossRef]

24. Oliveira, T.R.; Silva, C.M.; Macedo, D.F.; Nogueira, J.M.S. SNVC: Social networks for vehicular certification. Comput. Netw. 2016, 111, 129-140, doi:10.1016/j.comnet.2016.08.030. [CrossRef]

25. Silva, C.M.; Sarubbi, J.F.; Silva, D.F.; Porto, M.F.; Nunes, N.T. A Mixed Load Solution for the Rural School Bus Routing Problem. In Proceedings of the 2015 IEEE 18th International Conference on Intelligent Transportation Systems (ITSC), Las Palmas, Spain, 15-18 September 2015; pp. 1940-1945. [CrossRef]

26. Silva, C.M.; Guidoni, D.; Souza, F.S.; Pitangui, C.; Pitsillides, A. Using the Inter-Contact Time for Planning the Distribution of Roadside Units in Vehicular Networks. In Proceedings of the 19th IEEE International Conference on Intelligent Transportation Systems (ITSC 2016), Rio de Janeiro, Brazil, 1-4 November 2016.

27. Sarubbi, J.F.M.; Mesquita, C.M.R.; Wanner, E.F.; Santos, V.F.; Silva, C.M. A strategy for clustering students minimizing the number of bus stops for solving the school bus routing problem. In Proceedings of the NOMS 2016-2016 IEEE/IFIP Network Operations and Management Symposium, Istanbul, Turkey, 25-29 April 2016; pp. 1175-1180. [CrossRef]

28. Silva, C.M.; Meira, W., Jr. Design of roadside communication infrastructure with QoS guarantees. In Proceedings of the 2015 IEEE Symposium on Computers and Communication (ISCC), Larnaca, Cyprus, 6-9 July 2015; pp. 439-444. [CrossRef]

29. Silva, C.M.; Guidoni, D.L.; Souza, F.S.H.; Pitangui, C.G.; Sarubbi, J.F.M.; Pitsillides, A. Gamma Deployment: Designing the Communication Infrastructure in Vehicular Networks Assuring Guarantees on the V2I Inter-Contact Time. In Proceedings of the 2016 IEEE 13th International Conference on Mobile Ad Hoc and Sensor Systems (MASS), Brasilia, Brazil, 10-13 October 2016; pp. 263-271. [CrossRef]

30. Laplante, P. "Smarter" Roads and Highways. IEEE Internet Things Mag. 2018, 1, 30-35. [CrossRef]

31. Vegni, A.M.; Loscrí, V. A Survey on Vehicular Social Networks. IEEE Commun. Surv. Tutor. 2015, 17, 2397-2419. [CrossRef]

32. Basar, E.; Di Renzo, M.; De Rosny, J.; Debbah, M.; Alouini, M.; Zhang, R. Wireless Communications Through Reconfigurable Intelligent Surfaces. IEEE Access 2019, 7, 116753-116773. [CrossRef]

33. Renzo, M.D.; Debbah, M.; Huy, D.T.P.; Zappone, A.; Alouini, M.; Yuen, C.; Sciancalepore, V.; Alexandropoulos, G.C.; Hoydis, J.; Gacanin, H.; et al. Smart Radio Environments Empowered by AI Reconfigurable Meta-Surfaces: An Idea Whose Time Has Come. arXiv 2019, arXiv:1903.08925.

34. Bukhari, S.; Vardaxoglou, J.; Whittow, W. A Metasurfaces Review: Definitions and Applications. Appl. Sci. 2019, 9, 2727. [CrossRef]

35. Liaskos, C.; Nie, S.; Tsioliaridou, A.; Pitsillides, A.; Ioannidis, S.; Akyildiz, I. A New Wireless Communication Paradigm through Software-Controlled Metasurfaces. IEEE Commun. Mag. 2018, 56, 162-169. [CrossRef]

36. Liaskos, C.; Nie, S.; Tsioliaridou, A.; Pitsillides, A.; Ioannidis, S.; Akyildiz, I.F. Realizing Wireless Communication through Software-defined HyperSurface Environments. arXiv 2018, arXiv:1903.08925.

37. Liaskos, C.; Tsioliaridou, A.; Pitsillides, A.; Ioannidis, S.; Akyildiz, I.F. Using any Surface to Realize a New Paradigm for Wireless Communications. arXiv 2018, arXiv:1903.08925.

38. Tan, X.; Sun, Z.; Jornet, J.M.; Pados, D. Increasing indoor spectrum sharing capacity using smart reflect-array. In Proceedings of the 2016 IEEE International Conference on Communications (ICC), Kuala Lumpur, Malaysia, 22-27 May 2016; pp. 1-6. [CrossRef]

39. Liu, F.; Guo, J.; Zhao, L.; Shen, X.; Yin, Y. A Meta-Surface Decoupling Method for Two Linear Polarized Antenna Array in Sub-6 GHz Base Station Applications. IEEE Access 2019, 7, 2759-2768. [CrossRef]

40. Wu, Q.; Zhang, R. Intelligent Reflecting Surface Enhanced Wireless Network: Joint Active and Passive Beamforming Design. arXiv 2018, arXiv:1903.08925. 
41. Huang, C.; Zappone, A.; Alexandropoulos, G.; Debbah, M.; Yuen, C. Large Intelligent Surfaces for Energy Efficiency in Wireless Communication. 2018. Available online: https://deepai.org/publication/largeintelligent-surfaces-for-energy-efficiency-in-wireless-communication (accessed on 1 March 2020).

42. C-V2X Plugtest. Available online: https://www.etsi.org/events/1659-cv2x-plugtests\#pane-1/ (accessed on 1 March 2020).

43. Fallah, Y.P.; Huang, C.; Sengupta, R.; Krishnan, H. Analysis of Information Dissemination in Vehicular Ad-Hoc Networks With Application to Cooperative Vehicle Safety Systems. IEEE Trans. Veh. Technol. 2011, 60, 233-247. [CrossRef]

44. Balador, A.; Bohm, A.; Calafate, C.T.; Cano, J. A reliable token-based MAC protocol for V2V communication in urban VANET. In Proceedings of the 2016 IEEE 27th Annual International Symposium on Personal, Indoor, and Mobile Radio Communications (PIMRC), Valencia, Spain, 4-7 September 2016; pp. 1-6. [CrossRef]

45. Bazzi, A.; Campolo, C.; Masini, B.M.; Molinaro, A.; Zanella, A.; Berthet, A.O. Enhancing Cooperative Driving in IEEE 802.11 Vehicular Networks Through Full-Duplex Radios. IEEE Trans. Wirel. Commun. 2018, 17, 2402-2416. [CrossRef]

46. Bazzi, A.; Cecchini, G.; Zanella, A.; Masini, B.M. Study of the Impact of PHY and MAC Parameters in 3GPP C-V2V Mode 4. IEEE Access 2018. [CrossRef]

47. NXP USA, I. A of the Comments of NXP USA, Inc., in the Letter before the Federal Communications Commission, Washington, DC. 2019. Available online: https://autoalliance.org/wp-content/uploads/2017/ 01/5.9-GHz-Comments.pdf (accessed on 1 March 2020).

48. Sarubbi, J.F.M.; Silva, T.R.; Martins, F.V.C.; Wanner, E.F.; Silva, C.M. Allocating Roadside Units in VANETs Using a Variable Neighborhood Search Strategy. In Proceedings of the 2017 IEEE 85th Vehicular Technology Conference (VTC Spring), Sydney, Australia, 4-7 June 2017; pp. 1-5. [CrossRef]

49. Naik, G.; Choudhury, B.; Park, J. IEEE 802.11bd \& 5G NR V2X: Evolution of Radio Access Technologies for V2X Communications. arXiv 2019, arXiv:1903.08925.

50. Loscrí, V.; Natalizio, E.; Costanzo, C. Simulations of the Impact of Controlled Mobility for Routing Protocols. EURASIP J. Wirel. Commun. Netw. 2009, 2010, 315381. [CrossRef]

51. Chen, H.T.; Taylor, A.; Yu, N. A review of metasurfaces: Physics and applications. Rep. Prog. Phys. 2016, 79. [CrossRef]

52. Nadeem, Q.U.A.; Kammoun, A.; Chaaban, A.; Debbah, R.; Alouini, M.S. Intelligent Reflecting Surface Assisted Multi-User MISO Communication. arXiv 2019, arXiv:1906.02360.

53. A European strategy on Cooperative Intelligent Transport Systems, a Milestone towards Cooperative, Connected and Automated Mobility. 2016. Available online: https:/ / ec.europa.eu/transport/themes/its/cits_en (accessed on 1 Mar. 2020).

54. Hasan, S.; Balador, A.; Girs, S.; Uhlemann, E. Towards Emergency Braking as a Fail-Safe State in Platooning: A Simulative Approach. In Proceedings of the 2019 IEEE 90th Vehicular Technology Conference (VTC2019-Fall), Honolulu, HI, USA, 22-25 September 2019; pp. 1-5. [CrossRef]

55. Ammoun, S.; Nashashibi, F.; Laurgeau, C. An analysis of the lane changing manoeuvre on roads: The contribution of inter-vehicle cooperation via communication. In Proceedings of the 2007 IEEE Intelligent Vehicles Symposium, Istanbul, Turkey, 13-15 June 2007; pp. 1095-1100. [CrossRef]

56. Kim, S.; Qin, B.; Chong, Z.J.; Shen, X.; Liu, W.; Ang, M.H.; Frazzoli, E.; Rus, D. Multivehicle Cooperative Driving Using Cooperative Perception: Design and Experimental Validation. IEEE Trans. Intell. Transp. Syst. 2015, 16, 663-680. [CrossRef]

57. Wang, N.; Wang, X.; Palacharla, P.; Ikeuchi, T. Cooperative autonomous driving for traffic congestion avoidance through vehicle-to-vehicle communications. In Proceedings of the 2017 IEEE Vehicular Networking Conference (VNC), Amsterdam, The Netherlands, 14-16 November 2011; pp. 327-330. [CrossRef]

58. U.S. Department of Transportation (DOT), NHTSA Research Note.2018 Fatal Motor Vehicle Crashes Overview; U.S. Department of Transportation Report; NHTSA's National Center for Statistics and Analysis 1200 New Jersey Avenue SE: Washington, DC, USA, 2019.

59. Hussein, A.; Garcia, F.; Armingol, J.M.; Olaverri-Monreal, C. P2V and V2P communication for Pedestrian warning on the basis of Autonomous Vehicles. In Proceedings of the 2016 IEEE 19th International Conference on Intelligent Transportation Systems (ITSC), Rio de Janeiro, Brazil, 1-4 November 2016; pp. 2034-2039. [CrossRef] 
60. Wu, X.; Miucic, R.; Yang, S.; Al-Stouhi, S.; Misener, J.; Bai, S.; Chan, W. Cars Talk to Phones: A DSRC Based Vehicle-Pedestrian Safety System. In Proceedings of the 2014 IEEE 80th Vehicular Technology Conference (VTC2014-Fall), Vancouver, WC, Canada, 14-17 September 2014; pp. 1-7. [CrossRef]

61. Tahmasbi-Sarvestani, A.; Nourkhiz Mahjoub, H.; Fallah, Y.P.; Moradi-Pari, E.; Abuchaar, O. Implementation and Evaluation of a Cooperative Vehicle-to-Pedestrian Safety Application. IEEE Intell. Transp. Syst. Mag. 2017, 9, 62-75. [CrossRef]

62. Anaya, J.J.; Merdrignac, P.; Shagdar, O.; Nashashibi, F.; Naranjo, J.E. Vehicle to pedestrian communications for protection of vulnerable road users. In Proceedings of the 2014 IEEE Intelligent Vehicles Symposium Proceedings, Dearborn, MI, USA, 8-11 June 2014; pp. 1037-1042. [CrossRef]

63. Merdrignac, P.; Shagdar, O.; Nashashibi, F. Fusion of Perception and V2P Communication Systems for the Safety of Vulnerable Road Users. IEEE Trans. Intell. Transp. Syst. 2017, 18, 1740-1751. [CrossRef]

64. Sewalkar, P.; Krug, S.; Seitz, J. Towards 802.11p-based vehicle-to-pedestrian communication for crash prevention systems. In Proceedings of the 2017 9th International Congress on Ultra Modern Telecommunications and Control Systems and Workshops (ICUMT), Munich, Germany, 6-8 November 2017; pp. 404-409. [CrossRef]

65. Sewalkar, P.; Seitz, J. Vehicle-to-Pedestrian Communication for Vulnerable Road Users: Survey, Design Considerations, and Challenges. Sensors 2019, 19, 358. [CrossRef] [PubMed]

66. Themann, P.; Kotte, J.; Raudszus, D.; Eckstein, L. Impact of positioning uncertainty of vulnerable road users on risk minimization in collision avoidance systems. In Proceedings of the 2015 IEEE Intelligent Vehicles Symposium (IV), Seoul, South Korea, 29 June-1 July 2015; pp. 1201-1206. [CrossRef]

67. Kaiwartya, O.; Cao, Y.; Lloret, J.; Kumar, S.; Aslam, N.; Kharel, R.; Abdullah, A.H.; Shah, R.R. Geometry-Based Localization for GPS Outage in Vehicular Cyber Physical Systems. IEEE Trans. Veh. Technol. 2018, 67, 3800-3812. [CrossRef]

68. Wymeersch, H.; Seco-Granados, G.; Destino, G.; Dardari, D.; Tufvesson, F. 5G mmWave Positioning for Vehicular Networks. IEEE Wirel. Commun. 2017, 24, 80-86. [CrossRef]

69. Bagheri, M.; Siekkinen, M.; Nurminen, J.K. Cellular-based vehicle to pedestrian (V2P) adaptive communication for collision avoidance. In Proceedings of the 2014 International Conference on Connected Vehicles and Expo (ICCVE), Vienna, Austria, 3-7 November 2014; pp. 450-456. [CrossRef]

70. Yahiaoui, S.; Omar, M.; Bouabdallah, A.; Natalizio, E.; Challal, Y. An energy efficient and QoS aware routing protocol for wireless sensor and actuator networks. AEU Int. J. Electron. Commun. 2018, 83, $193-203$. [CrossRef]

71. Khairnar, V.; Kotecha, K. Performance of Vehicle-to-Vehicle Communication using IEEE 802.11p in Vehicular Ad-hoc Network Environment. Int. J. Netw. Secur. Its Appl. 2013, 5. [CrossRef] 